Proceedings of the 43rd "Jaszowiec", International School and Conference on the Physics of Semiconductors, Wisła 2014

\title{
Crystal Orientation Dependence of the Fundamental Optical Transition in type-II W-Design Quantum Well Structures
}

\author{
K. RYCZKO
}

Institute of Physics, Wrocław University of Technology, Wybrzeże Wyspiańskiego 27, 50-370 Wrocław, Poland

\begin{abstract}
Using a multiband $\boldsymbol{k} \cdot \boldsymbol{p}$ theory the band structure properties of type-II W-design $\mathrm{AlSb} / \mathrm{InAs} / \mathrm{GaInSb} / \mathrm{InAs} / \mathrm{AlSb}$ quantum wells on GaSb substrates of various crystallographic orientations have been investigated. Such structures are predicted for the emission in a broad range of mid infrared from below $3 \mu \mathrm{m}$ to beyond $10 \mu \mathrm{m}$. The energy of the fundamental optical transition and the corresponding oscillator strength have been determined in function of the layer structure details and versus the substrate orientation. In addition, the resulting optical anisotropy in such type-II quantum wells has been derived.
\end{abstract}

DOI: $10.12693 /$ APhysPolA.126.1149

PACS: 73.21.Fg, 71.55.Eq

\section{Introduction}

Semiconductor lasers emitting at wavelengths longer than $2-3 \mu \mathrm{m}$ are of great interest for applications in environmental pollution monitoring, medical diagnostics, infrared countermeasures, laser surgery and gas leakage detection [1-3]. In this wavelength region, there exist at least several competing concepts of sources of coherent radiation, including common laser diodes based on typeI quantum wells (QWs), quantum cascade lasers (QCLs) and interband cascade lasers (ICLs). For the latter ones, there have already been reported several advantageous features like broad tuning range of the emission, minimized influence of the Auger related carrier losses, and a very low power consumption [4]. However, in order to realize that, many parameters of these multilayer structures must be optimized, especially on the side of the active region which is composed of a cascade of type-II QWs made of a broken gap materials.

In this work, I consider type-II QWs in a form of the so called W-design, i.e. where a double potential well is used for the confinement of electrons, and hence the well energetic profile resembles the "W" letter [5]. We calculate the fundamental electronic structure properties of $\mathrm{AlSb} / \mathrm{InAs} / \mathrm{GaInSb} / \mathrm{InAs} / \mathrm{AlSb} \mathrm{QWs}$ grown on $\mathrm{GaSb}$ substrate on various crystallographic planes taking into account the effect of modifications in the band structure due to the related strain and piezoelectric field. The calculations are carried out within the eight-band $\boldsymbol{k} \cdot \boldsymbol{p}$ theory. We demonstrate the broad range of spectral tunability via the structure parameters modifications. We find that the fundamental $\mathrm{e}_{1}-\mathrm{hh} \mathrm{h}_{1}$ transition energy decreases as the crystal orientation is inclined from [001] toward [110]. We show that the transition energy $\mathrm{e}_{1}-\mathrm{hh}_{1}$ can still be efficiently optimized for wavelengths even beyond $8 \mu \mathrm{m}$, for a given range of thicknesses of InAs layers confining electrons, and for particular orientations of the substrate. Besides, as originally proposed by Yang [6] regarding the use of the type-II design, the broken gap structure of InAs-GaInSb leads to a partial local separation of electrons and holes and hence to significantly reduced transition oscillator strengths compared to typeI laser diode. Therefore, this issue has also been included in our considerations.

As crystal orientation modification induces anisotropies, we also calculate the degree of optical anisotropy in a QW structure of a W-shape confinement potential of this GaSb-based material system, which has not been reported for this kind of structures so far. There exist experimental data on in-plane optical anisotropy of type-II QWs but of $\mathrm{ZnTe} / \mathrm{CdSe}$ material system [7], and only in the case when these structures were grown on [001] GaAs substrate.

\section{Theoretical framework}

To calculate the electronic structure in the type-II Wdesign QW we use $8 \times 8 \mathrm{kp}$ Hamiltonians for the [001] growth direction $[8,9]$. The $[11 \mathrm{~N}]$-oriented Hamiltonian can be derived from the [001]-oriented one by using the transformation equations after Ref. [10]. The relation between the coordinate systems for the vector $\boldsymbol{k}$ are expressed as $\boldsymbol{k}=O \boldsymbol{k}^{\prime}$, with a rotation matrix $O$

$$
O=\left[\begin{array}{ccc}
\sin (\theta) / \sqrt{2} & -1 / \sqrt{2} & \cos (\theta) / \sqrt{2} \\
\sin (\theta) / \sqrt{2} & 1 / \sqrt{2} & \cos (\theta) / \sqrt{2} \\
-\cos (\theta) & 0 & \sin (\theta)
\end{array}\right],
$$

in which $\theta$ is the angle between the $k_{z}^{\prime}$ axis (parallel to the growth direction) and $k_{x}^{\prime}-k_{y}^{\prime}$ plane. For $\theta=0^{\circ}$ we have the [110]-oriented Hamiltonian and for $\theta=90^{\circ}$ we get the [001]-oriented Hamiltonian. For strained materials, the Bir-Pikus Hamiltonian [11] must be taken into consideration. In order to calculate the elastic stiffness coefficients and the components of the strain tensor in the new crystal orientation, the transformation matrix $O$ must be used together with the rules of tensor algebra. This is a straightforward exercise in algebraic manipula- 
tions which isdescribed in Ref. [12], hence not repeated here. The III-V compound semiconductors are piezoelectric materials. Once the off-diagonal strain components are known, one can then proceed to calculate the straininduced electric polarization in any layer

$$
P_{i}=2 e_{14} \varepsilon_{j k},
$$

where $e_{14}$ is the piezoelectric coefficient and $\varepsilon_{j k}$ is the strain tensor. The strain-induced polarization along the $[11 \mathrm{~N}]$ growth direction is then given by

$$
P=-\frac{2 e_{14}}{\sqrt{2+N^{2}}}\left(N \varepsilon_{x y}+\varepsilon_{y z}+\varepsilon_{z x}\right) .
$$

The polarization induced electric field along the [11N] growth direction of the $s$-th layer is given by [13]:

$$
E_{s}=\frac{\sum_{t}\left(P_{t}-P_{s}\right) \frac{L_{t}}{\epsilon_{t}}}{\epsilon_{s} \sum_{t} \frac{L_{t}}{\epsilon_{t}}},
$$

where $\epsilon$ is the dielectric constant and $L_{t}$ is the thickness of layer $t$.

In general, the carrier wave functions and the subband energies can be determined by numerically solving the Schrödinger equation using the finite difference method [14]. Knowing the energy band dispersion relations and the associated multicomponent wave functions, the momentum matrix element is obtained as in Ref. [15]. Then, the squared optical matrix element at the zone centre for light polarized in any direction can be calculated as

$$
|M|^{2}=|\langle\boldsymbol{c}|\boldsymbol{e} \cdot \hat{\boldsymbol{p}}| \boldsymbol{v}\rangle|^{2},
$$

where $|\boldsymbol{c}\rangle$ and $|\boldsymbol{v}\rangle$ are wave functions of the conduction and valence bands, respectively, $\boldsymbol{e}$ is the unit polarization vector light, $\hat{\boldsymbol{p}}$ is the momentum operator. I calculated the matrix elements between the lowest subbands of conduction and valence bands only ( $e_{1} h_{1}$ transition). The in-plane optical anisotropy - $\rho-$ of the $[11 \mathrm{~N}]$ oriented heterostructures was obtained by

$$
\rho=\frac{\left|M_{x^{\prime}}\right|^{2}-\left|M_{y^{\prime}}\right|^{2}}{\left|M_{x^{\prime}}\right|^{2}+\left|M_{y^{\prime}}\right|^{2}},
$$

where $\left|M_{x^{\prime}}\right|^{2}$ and $\left|M_{y^{\prime}}\right|^{2}$ are the squared optical matrix elements for the light linearly polarized in the $x^{\prime}$ direction $([n n(2 \bar{m})])$ and $y^{\prime}$ direction $([\overline{1} 10])$, respectively.

All the material parameters were taken from Refs. [16-18] for $300 \mathrm{~K}$.

\section{Results and discussion}

The results were obtained for the type-II W-design $\mathrm{AlSb} / \mathrm{InAs} / \mathrm{Ga}_{0.70} \mathrm{In}_{0.30} \mathrm{Sb} / \mathrm{InAs} / \mathrm{AlSb} \mathrm{QW}$ on GaSb. The InAs and $\mathrm{Ga}_{0.70} \operatorname{In}_{0.30} \mathrm{Sb}$ layers thicknesses are 2.5 and $3.5 \mathrm{~nm}$, respectively. At first, we calculated the strain-induced electric field in the growth direction, as shown in Fig. 1. The strain-induced piezoelectric field appears only for growth directions other than [001] and [110]. For [001] substrate orientation, the offdiagonal strain tensor components $\varepsilon_{x y}, \varepsilon_{y z}$ and $\varepsilon_{z x}$ are zero and from Eq. (3) we can see that no polarization fields will be induced for this particular growth direction. Whereas for structures which are grown on the [110] surface, only the off-diagonal term of the strain tensor $\varepsilon_{x y}$

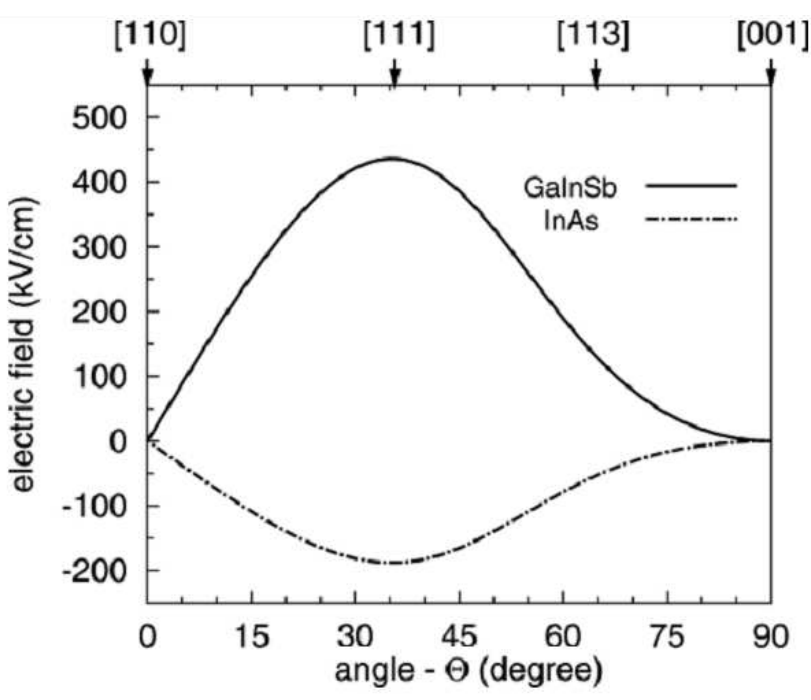

Fig. 1. The crystallographic orientation of the internal strain-induced piezoelectric field in the $\mathrm{Ga}_{0.70} \mathrm{In}_{0.30} \mathrm{Sb}$ (black solid line), InAs (black dash-dotted line).

is nonzero and hence some strain-induced piezoelectric field in the plane of the layer will exist. However, when considering the field in the [110] direction (i.e. along the growth axis for structures on the [110]-oriented substrates), it is obtained directly from Eq. (3) that no electric field is induced in that direction $(N=0)$ in Eq. (3). In contrast, the strain-induced piezoelectric field is the largest (in sense of its absolute value) in the InAs and $\mathrm{Ga}_{0 .} 7 \mathrm{I}_{n 0.3} \mathrm{Sb}$ regions for the structures oriented in the [111] growth direction. This is due to the fact that all three off-diagonal strain tensor components are equal and they sum up in Eq. (3).The electric field observed in the type-II W-design QW with a [111] crystal orientation is about $480 \mathrm{kV} / \mathrm{cm}$ (for the used In content of $30 \%$, giving the lattice mismatch of about $-1.9 \%$ compressive strain). This value is comparable to the magnitude estimated for [111]-oriented strained InGaAs on InP [19], although smaller than for [0001]-oriented wurtzite GaN/AlGaN QW structures [20].

Thus we expect that such a high electric field will affect significantly the conduction and valence band structure and the squared overlap integrals.

Figure $2 \mathrm{a}$ and $\mathrm{b}$ shows the conduction and valence heavy-hole band edge profiles, the fundamental electron $\mathrm{e}_{1}$ and heavy hole $\mathrm{hh}_{1}$ energy levels and the squared moduli of the corresponding wave functions (expressing the distribution of the probability densities), for two cases, respectively: without (a) and with (b) the electric polarization. This is shown for W-design QW structures with [113] substrate orientation. As expected, the maxima of the squared wave function moduli are in the corresponding layers of InAs and GaInSb for electrons and holes, respectively. However, from Fig. $2 \mathrm{a}$ and $\mathrm{b}$ one can see how the band structure is modified under the electric polarization, which further leads to an energy shift of the 

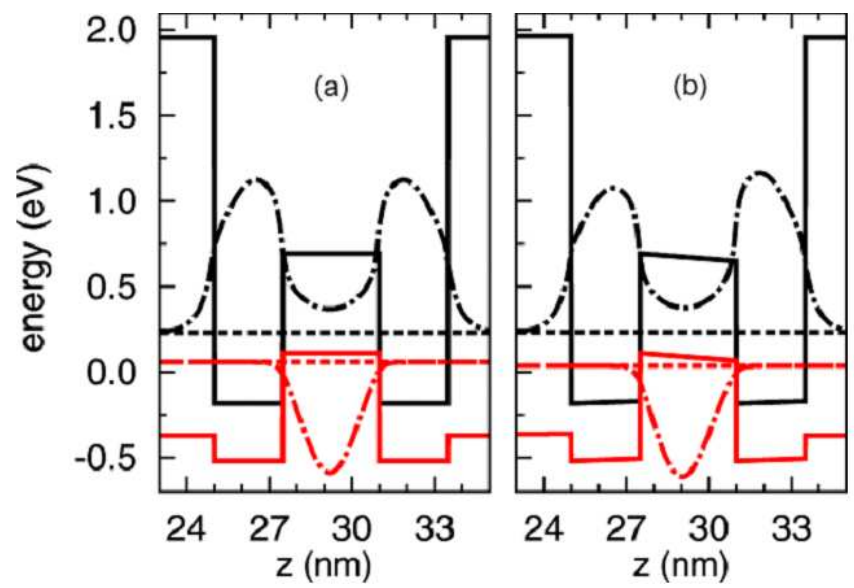

Fig. 2. Conduction (black solid line) and valence (red solid line) band edge lineup for type-II Wdesign $\mathrm{AlSb} / \mathrm{InAs} / \mathrm{Ga}_{0.7} \mathrm{In}_{0.3} \mathrm{Sb} / \mathrm{InAs} / \mathrm{AlSb} \mathrm{QW}$ for [113] growth direction; (a) without polarization effect, (b) with polarization effect. Dashed lines mark the lowest energy levels and dash-dotted lines represent the squared moduli of the wave functions of the fundamental electron $\mathrm{e}_{1}$ (black) and heavy hole $\mathrm{hh}_{1}$ (red) states.

electron and hole states by about $2 \mathrm{meV}$ and $21 \mathrm{meV}$, respectively. And hence, the fundamental $\mathrm{e}_{1}-\mathrm{hh}_{1}$ transition energy is increased accordingly.

In Fig. 3, the dependence of the transition energy and the corresponding squared wave functions overlap is shown in function of the InAs layer thickness for the type-II W-design QW on substrates of different orientations. In the range of the thickness of InAs layer changing from $1 \mathrm{~nm}$ up to $3 \mathrm{~nm}$ the $\mathrm{e}_{1}-\mathrm{hh}_{1}$ transition energy can shift in the corresponding wavelength range from below $3 \mu \mathrm{m}$ to above $10 \mu \mathrm{m}$, depending slightly on the chosen orientation. This is because the shape of the confinement potential and the electron effective mass is not changed, while the effective mass of holes in the valence subband changes, as shown in Table, when one deals with different substrate orientations. It can also be seen that, without the electric polarization effect, changing the growth direction leads to a change of the transition energy. When the polarization effect is taken into account, we obtain larger values of the $\mathrm{e}_{1}-\mathrm{hh}_{1}$ transition energy for [110] oriented substrate than for [001] substrate orientation. This is due to changes in the shape of the confinement potential in the conduction and valence bands under the electric field. Figure $3 \mathrm{~b}$ presents the corresponding squared wave functions overlap plotted in function of the InAs layer thickness for the W-design QW. It shows how significantly the changes in the confinement potential (via InAs layer thickness, or via the crystal orientation) affect the overlap. The effect of polarization is less significant, however when the polarization effect is included the maximum of the electron and hole probability density distribution is shifted in such a way that the overlap between the conduction and valence band states slightly increases.

\section{TABLE}

Effective mass of heavy hole in $\mathrm{Ga}_{0.70} \mathrm{In}_{0.30} \mathrm{Sb}$ for different substrate orientation in $\boldsymbol{k}^{\prime}=0.0 \mathrm{~cm}^{-1}$.

\begin{tabular}{c|c}
\hline \hline Crystal orientation & Mass of heavy hole $\left(m_{0}\right)$ \\
\hline$[001]$ & 0.254 \\
{$[110]$} & 0.371 \\
{$[113]$} & 0.363
\end{tabular}
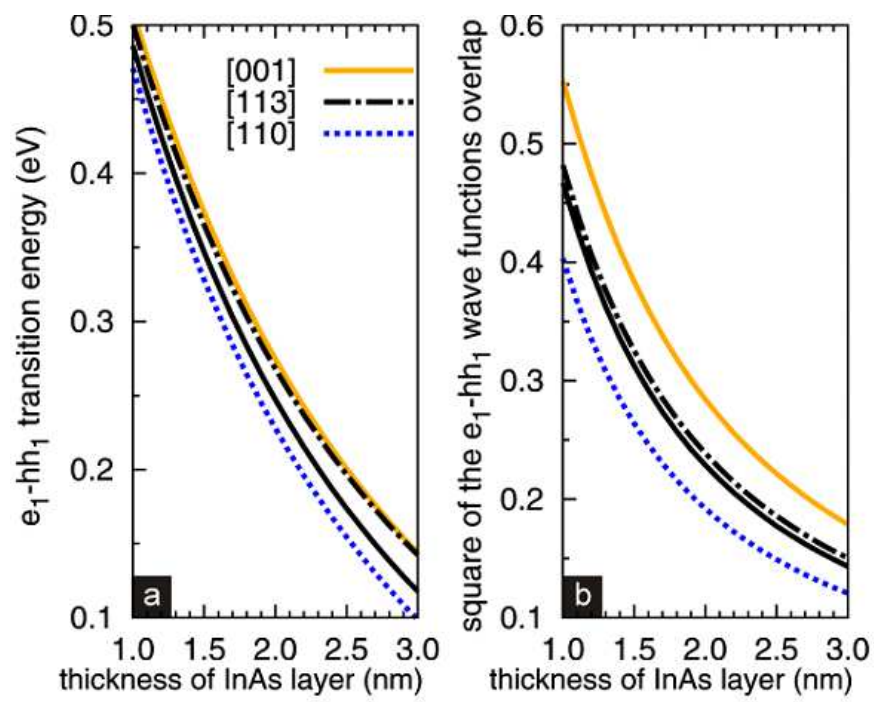

Fig. 3. The $\mathrm{e}_{1}-\mathrm{hh}_{1}$ transitions energy (a) and the squared wave functions overlap (b) in the type-II Wdesign AlSb/InAs $/ \mathrm{Ga}_{0.7} \operatorname{In}_{0.3} \mathrm{Sb} / \mathrm{InAs} / \mathrm{AlSb} \mathrm{QW}$ as a function of the InAs well width for three different growth direction: [001] (orange solid line), [113] (black dashdotted line - with polarization effect, black solid line — without polarization effect), [110] (blue dotted line).

Figure $4 \mathrm{a}$ and $\mathrm{b}$ shows the transition energy $\mathrm{e}_{1}-\mathrm{hh}_{1}$ and the corresponding squared wave functions overlap plotted as a function of the crystallographic orientation for the investigated W-design QW. There can mainly be observed that the piezoelectric effect is significant - for the [111] growth direction the obtained difference between the cases with and without the piezoelectric polarizaiton is about $80 \mathrm{meV}$. The squared overlap (Fig. 4b) experiences less the piezoelectricity but can change much with the crystallographic orientation - can increase by about $50 \%$ even.

In quantum wells on high-index substrates other than [001] and [111], the interband optical transition is anisotropic with respect to the polarization of light (absorbed or emitted). This requires deriving the optical anisotropy The resulting dependence of the optical anisotropy for compressively strained QW at $\boldsymbol{k}^{\prime}=0.0 \mathrm{~cm}^{-1}$, plotted in function of the substrate orientation, shows an interesting behaviour. The optical anisotropy of the $\mathrm{e}_{1}-\mathrm{hh}_{1}$ transition, for various In contents in the $\mathrm{Ga}_{1-x} \operatorname{In}_{x} \mathrm{Sb}$ layer and as a function of growth direction, is presented in Fig. 5. For all the compositions 


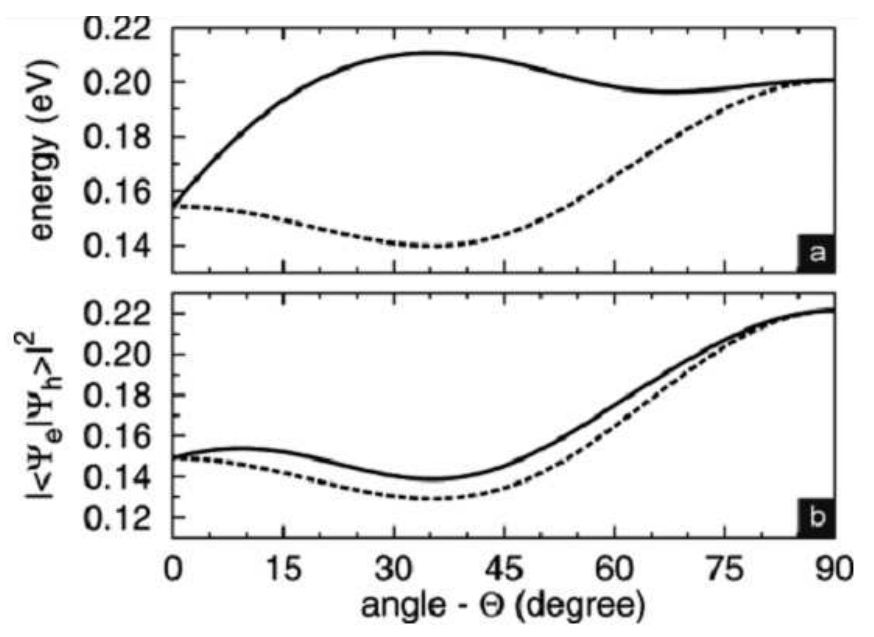

Fig. 4. (a) Transition energy $\mathrm{e}_{1}-\mathrm{hh}_{1}$ and (b) the square of the wave functions overlap, in the type-II W-design QW as a function of substrate orientation. Style lines mark the results of calculations with (solid) and without (dashed) the piezoelectric effect.

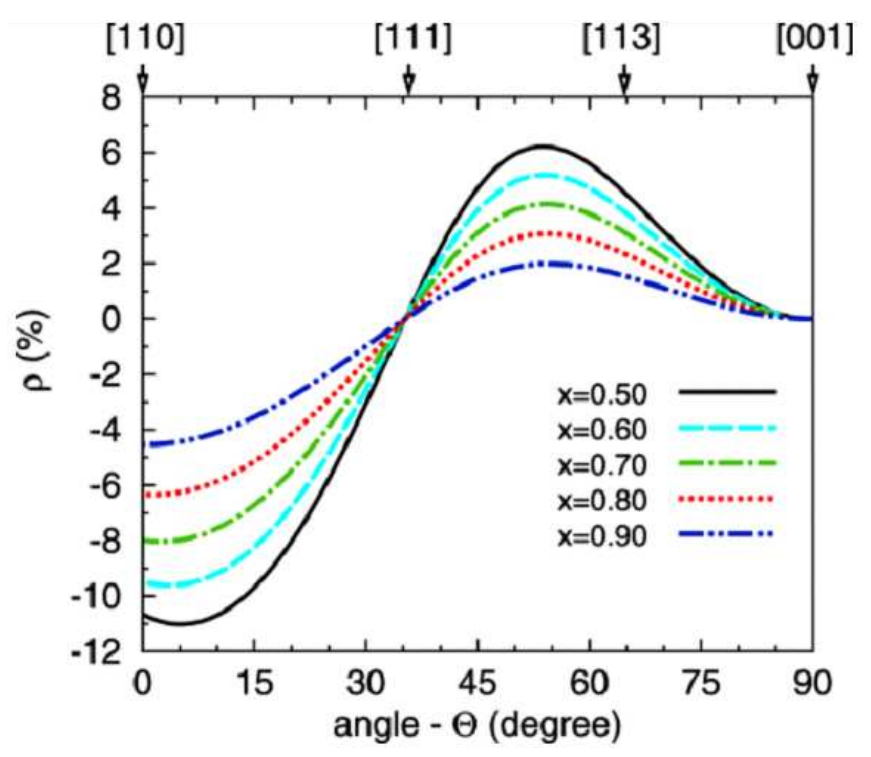

Fig. 5. Calculated degree of optical anisotropy for $\mathrm{e}_{1}-$ $\mathrm{hh}_{1}$ transition in the type-II W-design QW having various In contents in $\mathrm{Ga}_{1-x} \mathrm{In}_{x} \mathrm{Sb}$ layer, as a function of the substrate orientation.

there is no optical anisotropy in the QW on the [001] $\left(\theta=90^{\circ}\right)$ and [111] $\left(\theta=35^{\circ}\right)$ oriented substrates. Furthermore, the largest optical anisotropy occurs for [110] $\left(\theta=0^{\circ}\right)$ orientation. The optical anisotropy is positive when the growth direction of the $\mathrm{QW}$ is chosen between the [001] and [111] directions, which means that the preferential direction of polarization is $[11 \overline{2}]$. Whereas for a QW in which the substrate orientation is between the [111] and [110], the [110] is the preferential direction of polarization. This behaviour is consistent with that for more common type-I QWs [21, 22] (of other material systems). However, in type-II QWs the values of the optical anisotropy are smaller, by a factor of 2 at least than in similar QWs of type-I. Additionally, one can see that the increase in the In content influences the values of the optical anisotropy. There can mainly be observed that when the lattice mismatch is reduced, the absolute value of the optical anisotropy decreases.

\section{Conclusions}

In this work, I investigated the electronic structure of the type-II W-design AlSb/InAs/GaInSb/InAs/AlSb QW grown on GaSb substrate. I used the eight-band $\boldsymbol{k} \cdot \boldsymbol{p}$ theory with the effects of strain, crystal orientation and piezoelectric field included. It has been obtained that the fundamental $\mathrm{e}_{1}-\mathrm{hh}_{1}$ transition energy decreases as the crystal orientation is changed from [001] toward [110]. The absolute values of the internal piezoelectric fields in GaInSb layer are the largest for [111]-directed QW, and zero for the [001] and [110]-oriented QWs. Moreover, it was found that the preferential direction of the polarization changes when modifying the composition of the ternary layer confining the holes with increase of the In composition. I show that the transition energy $\mathrm{e}_{1}-\mathrm{hh}_{1}$ and its oscillator strength can still be efficiently optimized for wavelengths even beyond $8 \mu \mathrm{m}$, if the composition of the separating barrier, which is simultaneously the well for holes, is chosen properly, and for the given range of thicknesses of InAs layers confining electrons, for particular crystallographic orientation of the substrate.

\section{Acknowledgments}

This work was partly supported by the Project WideLase (No. 318798) of the 7-th Framework Programme of the European Commission. The author would also like to thank his colleagues, Jan Misiewicz and Grzegorz Sęk from Institute of Physics, Wrocław University of Technology, for fruitful discussions of the results, and for their valuable comments on this manuscript.

\section{References}

[1] N. Bandyopadhyay, Y. Bai, S. Tsao, S. Nida, S. Slivken, M. Razeghi, Appl. Phys. Lett. 101, 241110 (2012).

[2] Z. Tian, L. Li, H. Ye, R.Q. Yang, T.D. Mishima, M.B. Santos, M.B. Johnson, Electron. Lett. 48, 113 (2012).

[3] W.W. Bewley, C.L. Canedy, C.S. Kim, M. Kim, Ch.D. Merritt, J. Abell, I. Vurgaftman, J.R. Meyer, Opt. Express 20, 3235 (2012).

[4] I. Vurgaftman, W.W. Bewley, C.L. Canedy, C.S. Kim, M. Kim, C.D. Merritt, J. Abell, J.R. Lindle, J.R. Meyer, Nature Commun. 2, 585 (2011).

[5] J.R. Meyer, C.A. Hoffman, F.J. Bartoli, L.R. RamMohan, Appl. Phys. Lett. 67, 757 (1995).

[6] R.Q. Yang, Superlatt. Microstruct. 17, 77 (1995). 
[7] W.S. Su, M.H. Ya, Y.S. Chiu, Y.F. Chen, Phys. Rev. $B$ 66, 113305 (2002).

[8] H.R. Trebin, U. Rössler, R. Ranvaud, Phys. Rev. B 20, 686 (1979).

[9] K. Ryczko, G. Sęk, J. Misiewicz, J. Appl. Phys. 114, 223519 (2013).

[10] M. Kubisa, K. Ryczko, J. Misiewicz, Phys. Rev. B 83, 195324 (2011).

[11] L. Bir, G.E. Pikus, Symmetry and Strain-Induced Effects in Semiconductors, Wiley, New York 1974.

[12] J.M. Hinckley, J. Singh, Phys. Rev. B 42, 3546 (1990).

[13] F. Bernardini, V. Fiorentini, Phys. Status Solidi B 216, 391 (1999).

[14] J.W. Thomas, Numerical Partial Differential Equations, Finite Difference Methods, Springer, New York 1995.

[15] J.N. Schulman, Y.-C. Chang, Phys. Rev. B 31, 2056 (1985).
[16] I. Vurgaftman, J.R. Meyer, L.R. Ram-Mohan, J. Appl. Phys. 89, 5815 (2001).

[17] A. Beya-Wakata, P.-Y. Prodhomme, G. Bester, Phys. Rev. B 84, 195207 (2011).

[18] S. Adachi, Properties of Group-IV, III-V and II-VI Semiconductors, Wiley, Chichester 2005.

[19] E.A. Caridi, T.Y. Chang, K.W. Goossen, L.F. Eastman, Appl. Phys. Lett. 56, 659 (1990).

[20] S.H. Park, S.L. Chuang, Appl. Phys. Lett. 76, 1981 (2000).

[21] R. Winkler, A.I. Nesvizhskii, Phys. Rev. B 53, 9984 (1996).

[22] Y. Kajikawa, J. Appl. Phys. 86, 5663 (1999). 